\title{
Quantitative Analysis of Technical Polymer Mixtures by Matrix Assisted Laser Desorption/ Ionization Time of Flight Mass Spectrometry
}

\author{
Wenyan Yan \\ Bausch and Lomb, Inc., Rochester, New York, USA \\ Joseph A. Gardella, Jr. and Troy D. Wood \\ Department of Chemistry, University of Buffalo, The State University of New York, Buffalo, New York, USA
}

We report quantitative MALDI-TOF measurements for polydimethylsiloxane (PDMS) of two different molecular weights using the relative ratio of the signal intensities of integrated oligomer distributions for these two molecular weight distributions. By reporting the ratio of intensities of the integrals of two oligomer distributions, we assume that the ionization and desorption efficiencies, crystallization conditions and other factors affecting intensity are similar. Poly(methyl methacrylate) (PMMA-33,000) was mixed with PDMS samples to show whether the presence of another material might affect the desorption efficiency. Quantitative values for the number-average molecular weight $\left(M_{n}\right)$, weight-average molecular weight $\left(M_{w}\right)$ and polydispersities (D) were calculated using the oligomer distributions. The results show a linear relationship between the analyte concentrations and the signal intensities in the range from 1,000 Da to 10,000 Da, and the desorption efficiency of these two PDMS materials was the same even in the presence of PMMA. (J Am Soc Mass Spectrom 2002, 13, 914-920) (C) 2002 American Society for Mass Spectrometry

$\mathrm{T}$ To date, little experimental data has been presented illustrating the concept of quantitative analysis of low and high molecular weight compounds by use of matrix assisted laser desorption ionization mass spectrometry (MALDI-MS). The principal activity in this area has been reported by Nelson et al. [1] as well as by Hercules et al. [2-6]. In their studies, proteins and peptides were analyzed and the intensities of single peaks were considered. The quantitative information was calculated by comparing the signal intensities of analyte to the signal of internal standards. While MALDI has been applied to determine molecular weight characteristics of technical polymers [7], little effort has been made to deal with quantitative measurements for composition for technical polymers.

In the case of synthetic polymers, oligomer chains and subunits show a distribution of molecular weights. More recently, to obtain accurate measurement of molecular weights in polydisperse polymers, gel permeation chromatography (GPC) or size exclusion chromatography (SEC) have been coupled with mass spectrometry. The studies of Montaudo et al. [8] and Simonsick and co-workers [9] demonstrated that these

Published online June 13, 2002

Address reprint requests to Dr. J. A. Gardella, Jr., Department of Chemistry, University of Buffalo, The State University of New York, 470 Natural Sciences Complex, Buffalo, NY 14260-3000, USA. E-mail: gardella@acsu.buffalo.edu techniques were able to section the polydisperse polymers into several narrow polydispersity fractions, and accurate measurements of MW were obtained.

In the work of Nelson et al. [1], where MALDI-TOF was used for protein quantification, their results demonstrated a linear relationship of molecular ion signals to the concentration of protein in solution. This method used the normalization of analyte molecular ion signals to the signals of internal reference molecular ions.

The results for the concentration of proteins are somewhat different depending upon which method of internal reference standard was utilized. The protein concentrations determined from the calibration curves depended upon which method of internal reference standard was utilized.

In the first report of a quantitative measurement utilizing MALDI by Hercules and co-workers [2], this group studied cyclosporin A in blood by TOF-SIMS and MALDI-TOF. Cyclosporin A belongs to a group of nonpolar, cyclic oligopeptides that have immunosuppressant activity. It was found that quantitative data for cyclosporin A were obtained using both TOF-SIMS and MALDI-TOF. An internal standard was employed for both techniques. The limit of detection (LOD) was 7 $\mathrm{ng} / \mathrm{ml}$ and $10 \mathrm{ng} / \mathrm{ml}$ for TOF-SIMS and MALDI, respectively. Hercules et al. studied the direct coupling of thin-layer chromatography (TLC) with MALDI for the analysis of a cocaine hydrochloride, using cocaine- $\mathrm{d}_{3}$ as 
an internal standard [3]. Their rationale for combining TLC with MALDI was based upon TLC being used as a standard method for the analysis of drugs of abuse, while cocaine- $\mathrm{d}_{3}$ is available as an internal standard, and that quantitative analysis of cocaine by MALDI had been previously demonstrated [4]. These authors found that TLC coupled with MALDI demonstrated quantitative analysis with the use of cocaine hydrochloride as a model compound and cocaine- $\mathrm{d}_{3}$ as the internal standard. The relative standard deviation of the standard curve slope was found to be better than $3 \%$. The relative standard deviation of the analyte/internal standard intensity ratios was between 3.8 to $9.5 \%$. The precision was found to be better than $12 \%$, while the detection limit was approximately $60 \mathrm{pg}$. This work was later supported by additional studies of Hercules et al., using TLC-MALDI for the separation and detection of low molecular weight peptides, oligonucleotides, dyes, pesticides, and drugs of abuse [5].

Hercules and co-workers also reported quantitative information from analysis of peptides and proteins by using MALDI [6]. Different matrices were used in their study. The comparative results showed that multicomponent gentisic acid (2,5-dihydroxybenzoic acid [DHB]) and ferulic acid (4-hydroxy-3-methoxycinnamic acid [FA]) gave better accuracy, sensitivity, and linear response over a wide range for cyclosporin A $(8 \mathrm{fmol}$ to $1700 \mathrm{fmol}$ ) and insulin (0.1 to $100 \mathrm{pmol})$ than for several other commonly used matrices. Their studies also showed that the threshold of analyte/matrix molar ratio is $1 / 3000$ in order to obtain the best linearity of the standard curve and the accuracy of the quantitative analysis.

In a recent work by Siuzdak and co-workers, an automated MALDI and electrospray ionization (ESI) approach was used for the quantitative analysis of cyclosporin A, using cyclosporin $\mathrm{G}$ as an internal standard. The limits of detection were determined to be 15 $\mathrm{ng} / \mathrm{ml}$ in whole blood for ESI/MS and MALDI. The authors state that this approach has utility as a pharmacokinetic tool [10].

Because the absolute intensity of the MALDI signal strongly depends on the laser power and the matrix crystallization conditions [11], the use of an internal standard becomes essential. However, the use of an internal standard with different chemical properties from the analyte may result in different crystallization of the matrix, hence it may produce different MALDI response than the analyte, and requires a highly stable instrument as well as constant analyte/matrix molar ratios.

In the present study, quantitative measurements are demonstrated for the first time for polydimethylsiloxane (PDMS) of two different molecular weights using the relative ratio of the signal intensities of integrated oligomer distributions for these two molecular weight distributions. By reporting the ratio of intensities of the integrals of two oligomer distributions, we can assume that the ionization and desorption efficiencies, crystal-

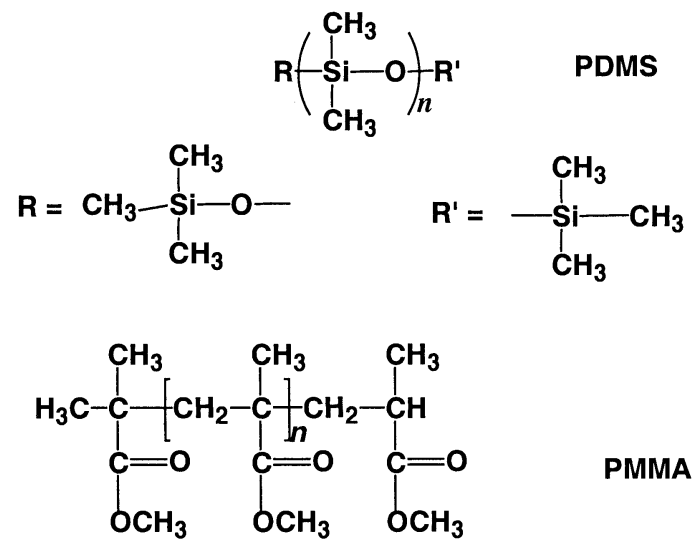

Scheme 1 Structures of polymers studied.

lization conditions, and other factors affecting intensity are similar. Poly(methyl methacrylate) (PMMA33,000) was mixed with PDMS samples to show whether the presence of another material might affect the desorption efficiency. The methods of sample preparation and data interpretation are discussed also. The number-average molecular weight $\left(\mathrm{M}_{\mathrm{n}}\right)$, weight-average molecular weight $\left(\mathrm{M}_{\mathrm{w}}\right)$ and polydispersities (D) were calculated using the oligomer distributions. The results show a linear relationship between the analyte concentrations and the signal intensities in the range from $1000 \mathrm{Da}$ to $10,000 \mathrm{Da}$, and the desorption efficiency of these two PDMS materials was the same even in the presence of PMMA.

\section{Experimental}

Mass spectrometry was carried out on a Voyager-DE STR (Applied Biosystems, Framingham, MA) which includes time-of-flight and a reflector analyzer. The linear drift length is $1.3 \mathrm{~m}$, which is modified for delayed extraction. The linear mode was chosen over the reflector mode to maximize signal intensity since resolving power was sufficient to differentiate the observed oligomers. The instrument is equipped with a standard nitrogen laser, $(\lambda=337 \mathrm{~mm}$, Laser Science International, Newton, MA) to desorb and ionize the samples. The principles and operation of the system are described elsewhere [12]. The instrument was operated in the positive ion linear mode with an accelerating potential of $+25 \mathrm{kV}$. The resolution $(\mathrm{m} / \Delta \mathrm{m}$ at fwhm) of the linear detector mode is about 800 . All spectra were collected at a average of 200 laser shots.

All samples were analyzed using 2,5-dihydroxybenzoic acid (2,5-DHB) (Aldrich Chemical Co., Inc., Milwaukee, WI) as the matrix. Scheme $\mathbf{1}$ shows the structures of the two materials studied in the present paper. The two PDMS samples (produced by anionic polymerization from n-butyl lithium initiation of cyclic D-3 monomers by Polymer Source, Inc., Dorval, Quebec, Canada) used in this study have average mass $M_{n} \sim$ $2200 \mathrm{Da}$ and $\mathrm{M}_{\mathrm{n}} \sim 6140 \mathrm{Da}$ (GPC results provided by 
Table 1. Ratios of PDMS and PMMA in the mixtures

\begin{tabular}{lcccc}
\hline & $\begin{array}{c}\text { PDMS6140 } \\
(\mu \mathrm{g})\end{array}$ & $\begin{array}{c}\text { PDMS2200 } \\
(\mu \mathrm{g})\end{array}$ & $\begin{array}{c}\text { PMMA } \\
(\mu \mathrm{g})\end{array}$ & $\begin{array}{c}\text { TOTAL } \\
(\mu \mathrm{g})\end{array}$ \\
\hline \hline Sample 1a & 50 & 10 & 40 & 100 \\
Sample 2a & 40 & 20 & 40 & 100 \\
Sample 3a & 30 & 30 & 40 & 100 \\
Sample 4a & 20 & 40 & 40 & 100 \\
Sample 5a & 10 & 50 & 40 & 100 \\
Sample 6a & 0 & 60 & 40 & 100 \\
\hline & & & & \\
& & & & \\
& & $(\mu \mathrm{g})$ & $(\mu \mathrm{g})$ & $(\mu \mathrm{g})$ \\
\hline Sample 1b & 50 & 50 & 0 & 100 \\
Sample 2b & 40 & 40 & 20 & 100 \\
Sample 3b & 30 & 30 & 40 & 100 \\
Sample 4b & 20 & 20 & 60 & 100 \\
Sample 5b & 10 & 10 & 80 & 100 \\
\hline
\end{tabular}

the manufacturer, $\mathrm{D}<1.1)$. PDMS polymerized using anionic conditions results in non-polar end group capping by trimethyl silane, assuring the mass structural assignments shown in Scheme 1. PMMA supplied as having average mass $\mathrm{Mn} \sim 33,000 \mathrm{Da}$ was obtained from Scientific Polymer Products, Inc., Ontario, NY. Both PDMS and PMMA were dissolved in chloroform at a concentration of $1 \mathrm{mg} / \mathrm{ml}$. Then various amounts of the solutions of different molecular weights of PDMS were mixed with the PMMA solution. Table 1 lists the ratio of PDMS2200, PDMS6140, and PMMA in each mixture. In set $\mathrm{A}$, the PMMA concentrations to total PDMS concentration were kept constant (40:60) while the concentrations of PDMS2200 and PDMS6140 were varied. In set $B$, the relative concentrations of PDMS2200 to PDMS6140 were kept constant (i.e., 50:50) and PMMA was varied from $0-80 \% .30 \mathrm{mg}$ of $2,5-\mathrm{DHB}$ was dissolved in $1 \mathrm{ml}$ of tetrahydrofuran (THF). $2 \mu \mathrm{l}$ of the matrix solution was deposited on the sample plate and dried for $5 \mathrm{~min} .1 \mu \mathrm{l}$ of the mixture solution then was deposited on the top of the dry matrix.

\section{Results and Discussion}

Laser power is a crucial parameter for spectral quality in MALDI. The previous work of Derrick and coworkers showed that increasing laser power significantly skewed the molecular weight distribution of PEO toward lower mass [13]. High laser power yields increases in signal intensity but increases the probability of obtaining fragment signals and saturating the detector. Low laser power results in a lack of sensitivity and peak distortion. In this study, it was important to choose an optimal laser power that can give the strongest signal intensity for both low and high mass PDMS. The molecular weight of matrix DHB is $154 \mathrm{Da}$. In order to reduce the detector saturation, the starting mass of all collected mass spectra was set to $500 \mathrm{Da}$. The results showed that lower laser power shows a significant signal loss of high molecular weight PDMS, while higher laser power increased the total signal counts, but caused the saturation of the detector. The baseline noise also increased. The laser power setting of 2200 on the Voyager data system was chosen as the optimal intensity for both $2 \mathrm{~K}$ and $6 \mathrm{~K}$ PDMS and was used throughout the study.

The relative ratio of analyte to matrix is another critical factor for successful MALDI analysis. The matrix serves to separate analyte molecules from each other, absorb laser energy and propel analyte molecules into the test chamber in the gas phase. If the matrix molecule concentration was less than was needed to separate all analyte molecules, the inter/intra molecule interaction among the analyte molecules might hinder the ionization/desorption efficiency. Furthermore, with low matrix concentration not enough laser energy is absorbed; both phenomena result in low signal counts. On the other hand, a large amount of matrix will dilute the overall analyte concentration, also causing low signal counts. It is suggested by the manufacturer that the polymer analyte concentration should be approximately $10^{-4} \mathrm{M}$ in order to obtain reasonable signal to noise [14]. Two different matrix concentrations (10 $\mathrm{mg} / \mathrm{ml} \mathrm{DHB}$ and $30 \mathrm{mg} / \mathrm{ml} \mathrm{DHB}$ ) were tested. The signals from the samples made from $10 \mathrm{mg} / \mathrm{ml}$ matrix were almost three times more intense than the signals of sample where the $30 \mathrm{mg} / \mathrm{ml}$ matrix was used. A higher percent of matrix usage was not attempted in this study. $10 \mathrm{mg} / \mathrm{ml}$ was chosen as the matrix concentration throughout the study.

Single component PDMS2200 and PDMS6140 samples in DHB were analyzed prior to the sample analysis of the mixtures. It was found that both PDMS2200 and PDMS6140 yielded a symmetric Gaussian-like distribution of peaks because of the protonated oligomers, as shown in Figure 1a and b. A three-peak repeat pattern was observed in both PDMS2200 (Figure 2) and PDMS6140 spectra similar to that reported previously for PDMS produced by anionic polymerization [15, 17]. The pattern results from ionization of oligomers formed from the three different initiators in the anionic polymerization conditions, with lithium silanates of one, two, and three monomer units forming from the ring opening of D-3 monomer [15]. For PDMS2200, every second oligomer showed a higher intensity than the other surrounding oligomer peaks, corresponding to the chain length of $3 n+1$. The oligomers corresponding to $3 n+2$ were slightly higher than the oligomer peaks corresponding to $3 \mathrm{n}$ chain length. Theoretically, $3 n$ oligomers should be the exclusive products and strongest peaks in the spectra since hexamethylcyclosilaxane was the monomer. The MALDI results indicate that redistribution of initiator species occurs during the polymerization process. The same patterns were also observed by TOF-SIMS, and other experiments have been conducted to investigate this issue [15]. For PDMS 6140 , every first oligomer showed the strongest intensity, corresponding to 3 n oligomer, as expected from the same polymerization process discussed before. The 


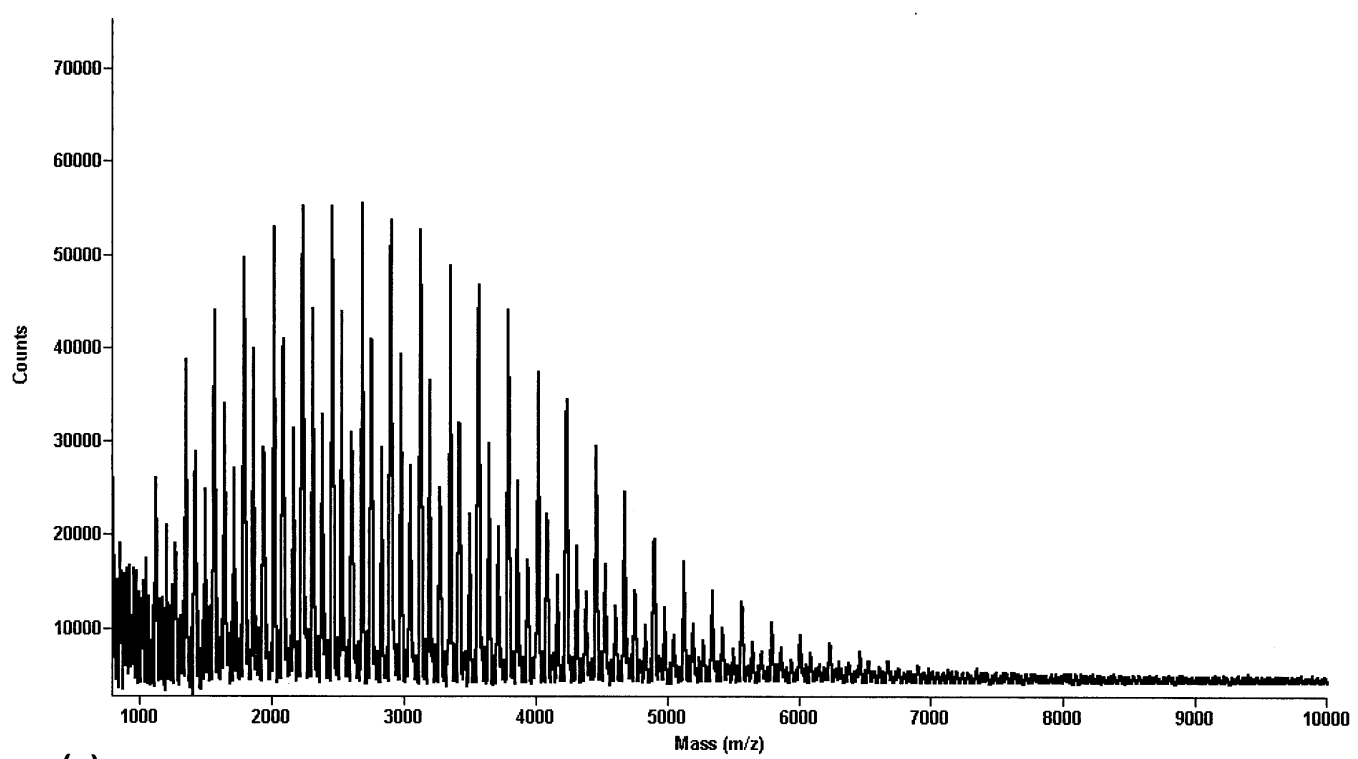

(a)

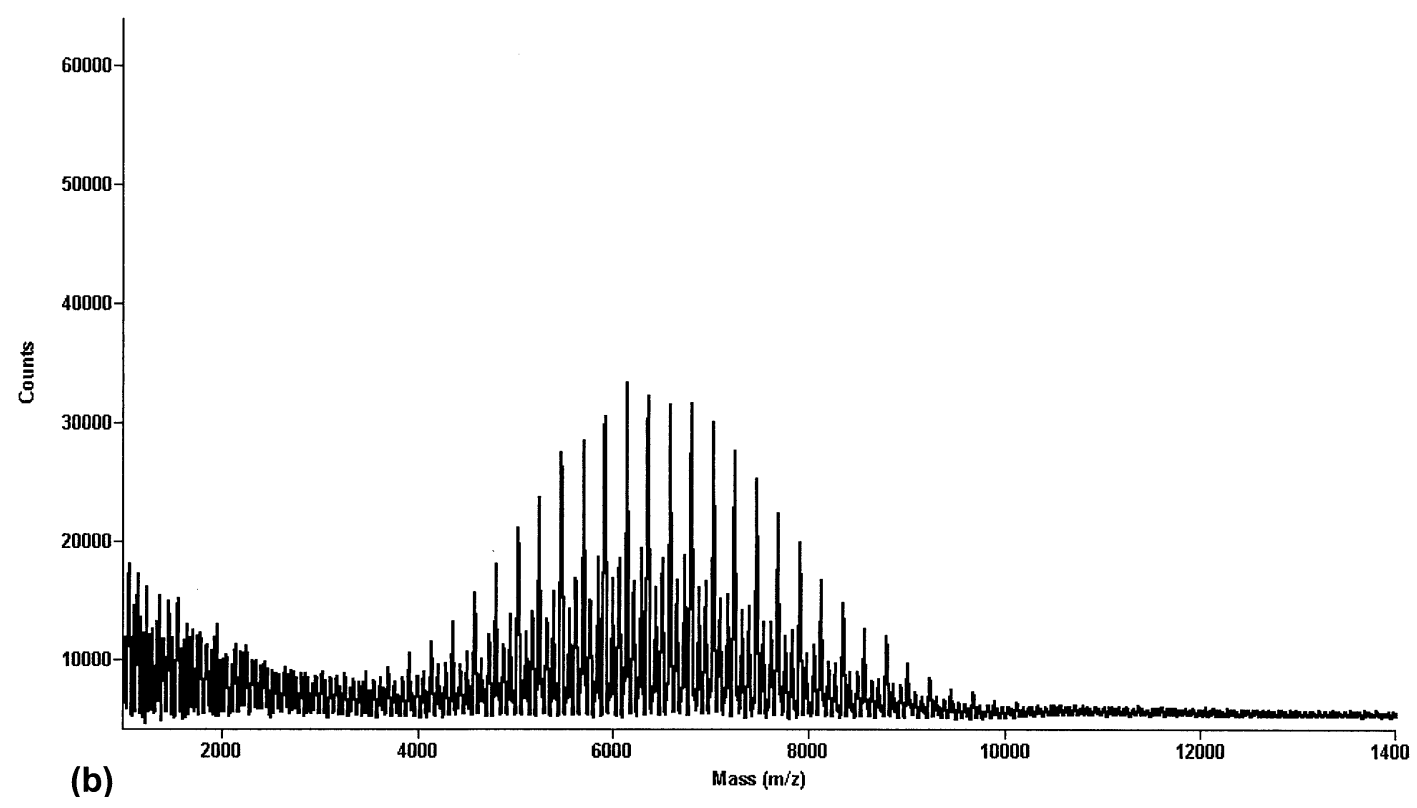

Figure 1. (a) MALDI spectrum of PDMS2200. (b) MALDI spectrum of PDMS6140.

mass difference between each peak is $74 \mathrm{Da}$, which equals the mass of a repeat unit of PDMS.

The molecular weights and their distribution of polymers are evaluated using the number-average molecular weight $\left(M_{n}\right)$, the weight-average molecular weight $\left(M_{w}\right)$ and polydispersities (D). $M_{n}$ is calculated from eq 1:

$$
M_{n}=\Sigma m_{i} n_{i} / \Sigma n_{i}
$$

where $m_{i}$ is the mass and $n_{i}$ is the intensity of the $i$ th oligomer in the distribution. $\mathrm{M}_{\mathrm{w}}$ is calculated from eq 2:

$$
M_{w}=\Sigma m_{i}^{2} n i / \Sigma m_{i} n_{i}
$$

polydispersity, $\mathrm{D}$, is defined by eq 3 :

$$
\mathrm{D}=\mathrm{M}_{\mathrm{w}} / \mathrm{M}_{\mathrm{n}}
$$

The $\mathrm{M}_{\mathrm{n}}, \mathrm{M}_{\mathrm{w}}$, and D of PDMS2200 and PDMS6140 were calculated using the Voyager GRAMS software. The calculation results and the information obtained from the manufacturer are listed in Table 2 for comparison.

The calculated data from MALDI showed that the molecular distribution overall is shifted to the higher mass for both PDMS2200 and PDMS6140 compared to the SEC data. The calculated values of PDMS6140 are in relatively good agreement with the SEC data $\left(\mathrm{M}_{\mathrm{n}}=\right.$ $6361 \pm 22, \mathrm{M}_{\mathrm{w}}=6845 \pm 27, \mathrm{D}=1.08 \pm 0.01$ for MALDI versus $M_{n}=6140, M_{w}=6660, D=1.07$ for SEC), more so than the calculated values of PDMS2200 $\left(\mathrm{M}_{\mathrm{n}}=2672\right.$ $\pm 147, \mathrm{M}_{\mathrm{w}}=3000 \pm 204, \mathrm{D}=1.13 \pm 0.02$ for MALDI versus $M_{n}=2200, M_{w}=2400, D=1.09$ for SEC). The 

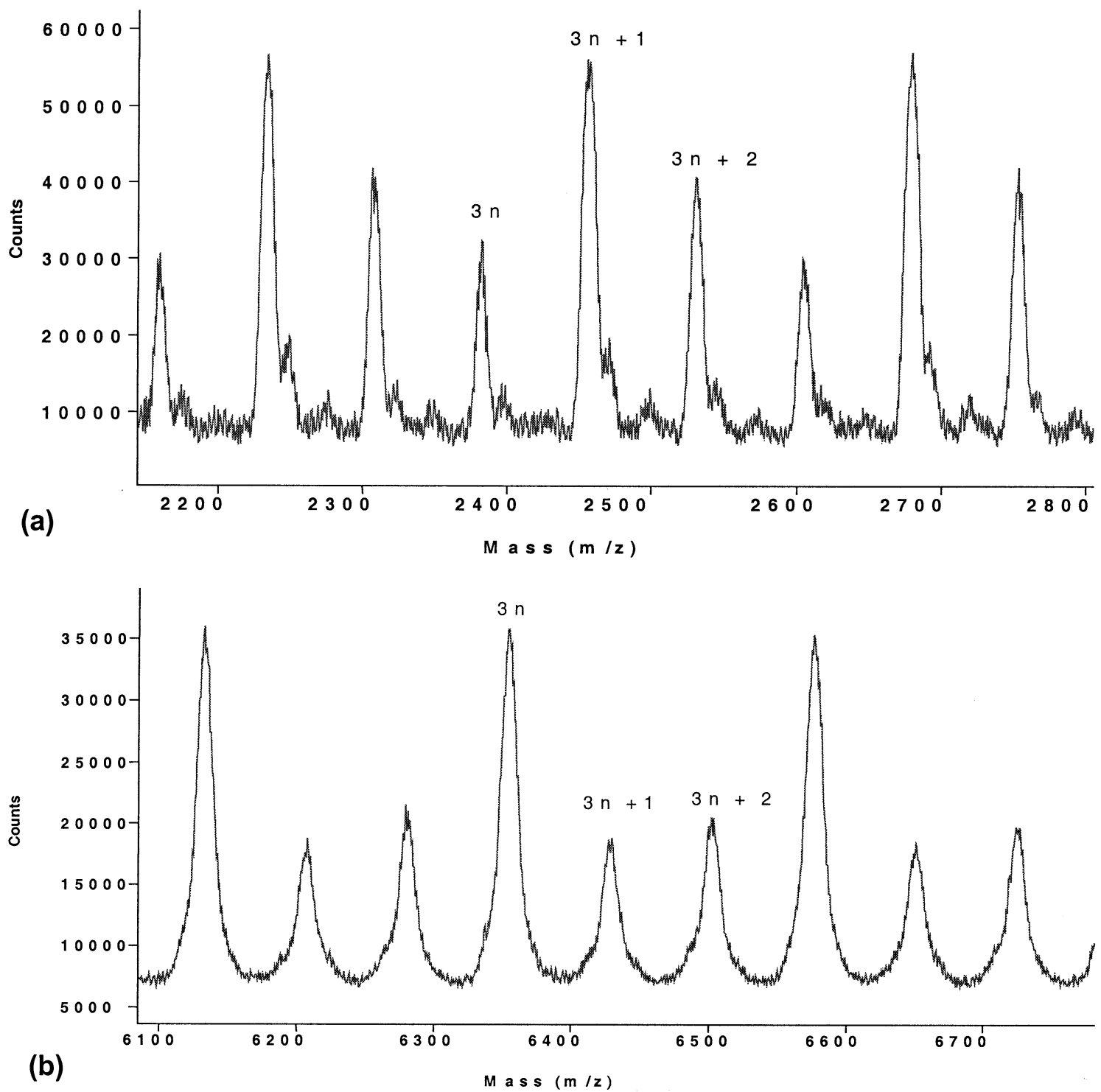

Figure 2. Repeat patterns in the MALDI spectrum of (a) PDMS2200 and (b) PDMS6140.

differences are likely due to discrimination against lower mass oligomers. Derrick and co-workers showed the existence of discrimination against the low-mass ions in MALDI compared to SEC [16]. This may be in part due to the higher volatility of low mass oligomers than high mass oligomers; it is well known that low mass oligomers of PDMS are liquidlike and have very low volatilities [17]. The vacuum system may in fact remove low mass oligomers preferentially. Schriemer and Li have studied the possible source of mass discrimination and concluded that both sample preparation and instrument factors could contribute to low mass discrimination [18]. Previous studies also showed that high polydispersities normally cause the disagreement between MALDI data and SEC measurements [19-21]. The uncertainties associated with MALDI TOF molecular weight distribution measurements for polystyrene have been extensively discussed in a round

Table 2. Calculation results of $\mathrm{M}_{\mathrm{n}}, \mathrm{M}_{\mathrm{w}}$, and polydispersity of PDMS

\begin{tabular}{lcccc}
\hline & $\begin{array}{c}\text { PDMS2200 } \\
\text { manufacturer } \\
\text { data* }\end{array}$ & $\begin{array}{c}\text { PDMS2200 } \\
\text { calculated } \\
\text { data }\end{array}$ & $\begin{array}{c}\text { PDMS6140 } \\
\text { manufacturer } \\
\text { data* }\end{array}$ & $\begin{array}{c}\text { PDMS6140 } \\
\text { calculated } \\
\text { data }\end{array}$ \\
\hline \hline $\mathrm{M}_{\mathrm{n}}$ & 2200 & $2672 \pm 147$ & 6140 & $6361 \pm 22$ \\
$\mathrm{M}_{\mathrm{w}}$ & 2400 & $3000 \pm 204$ & 6660 & $6845 \pm 27$ \\
Polydispersity & 1.09 & $1.13 \pm 0.02$ & 1.07 & $1.08 \pm 0.01$ \\
\hline
\end{tabular}

*Size exclusion chromatography (SEC) data supplied by the manufacturer. 


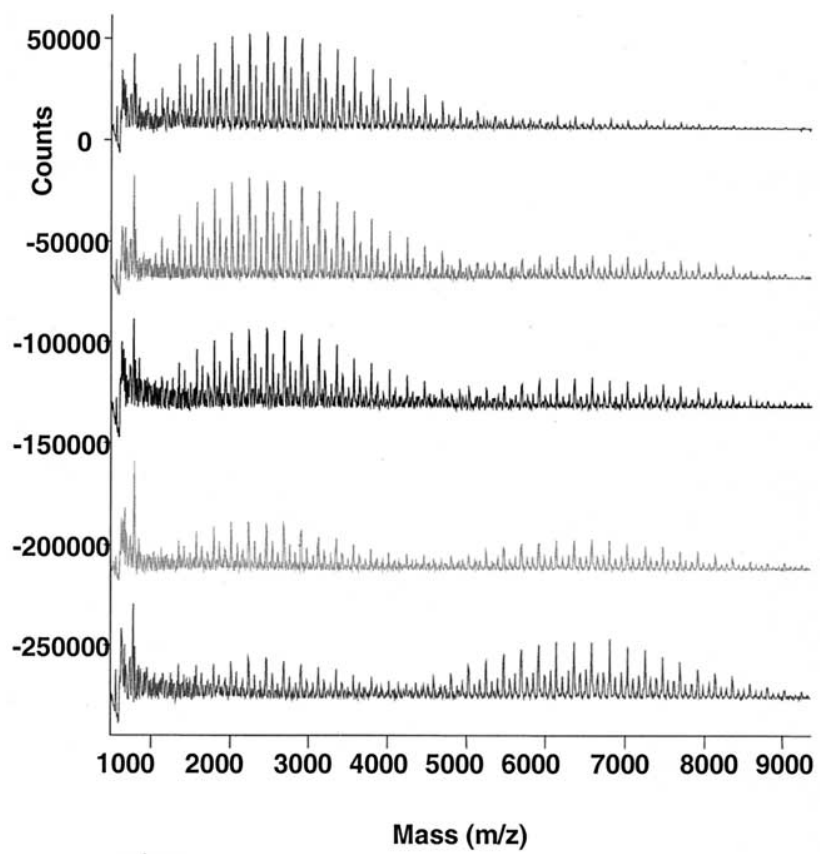

Figure 3. Stacked MALDI spectra of Sample 1, 2, 3, 4, 5.

robin study sponsored by NIST and recently reported in Analytical Chemistry [22]. Preliminary aspects of molecular weight analysis by MALDI have been explored [23]. MALDI experimental parameters can also affect the appearance of a copolymer mass distribution and skew MALDI compositional analysis according to monomers used in copolymer synthesis [24].

A series of samples were prepared containing the same amount of PMMA and different amounts of PDMS, listed in Table 1. The purpose of this series was to investigate whether different length oligomers display equal ionization probabilities. Molecular ion distributions were observed in each spectrum of the samples. PDMS2200 showed stronger absolute intensity than PDMS6140. The same type of repeat pattern as discussed earlier in this chapter was observed for both low and high molecular weight PDMS.

As shown in Figure 3, the signal intensities for both PDMS2200 and PDMS6140 increased with the increase of analyte concentrations. No signal from PMMA was detected; this is likely due to the extremely high polydispersity (e.g., $\mathrm{D}=$ ca. 3). Normally the MALDI technique permits an accurate determination of molecular weights for narrowly distributed polymers $(\mathrm{D}<1.2)$ [20]. With $\mathrm{D}$ higher than 1.5, it becomes difficult for determination.

In order to obtain accurate information, all oligomer intensities were summed to give the total signal intensity for the concentration of that particular molecular weight fraction. The total signal intensity of each polymer was calculated using the following eq:

$$
I_{t}=\Sigma I_{p}^{*}\left(\left(M_{p}-57-73\right) / 74\right)
$$

Where $I_{t}$ is the total signal intensity, $I_{p}$ is the intensity of each oligomer peak, $M_{p}$ is the mass of each oligomer peak,
Table 3. Comparison of signal intensity ratios to concentration ratios

\begin{tabular}{lccc}
\hline Name & $\begin{array}{c}\mathrm{V}_{6140} / \mathrm{V}_{2200} \\
(\mu \mathrm{l})\end{array}$ & $\begin{array}{c}\mathrm{M}_{6140} / \mathrm{M}_{2200} \\
\text { (theoretical) }\end{array}$ & $\begin{array}{c}\mathrm{I}_{\mathrm{t} / 2 \mathrm{k}} / \mathrm{I}_{\mathrm{t} / 6 \mathrm{k}} \\
\text { (calculated) }\end{array}$ \\
\hline \hline Sample 1 & $50 / 10$ & 5.0 & $4.72 \pm 0.86$ \\
Sample 2 & $40 / 20$ & 2.0 & $2.11 \pm 0.23$ \\
Sample 3 & $30 / 30$ & 1.0 & $0.97 \pm 0.05$ \\
Sample 4 & $20 / 40$ & 0.5 & $0.55 \pm 0.04$ \\
Sample 5 & $10 / 50$ & 0.2 & $0.21 \pm 0.03$ \\
Sample 6 & $0 / 60$ & 0.0 & $0.00 \pm 0.00$ \\
\hline
\end{tabular}

57 is the mass of the isobutyl end group on one side and 73 is the mass of the siloxane end group on the other side, and 74 is the mass of dimethylsiloxane repeat unit.

The weight concentrations of both PDMS sample solutions were $1 \mathrm{mg} / \mathrm{ml}$, which makes the total monomer molar concentration of the oligomer for both PDMS2200 and PDMS6600 the same. As is the practice in polymer science, the monomer moles directly calculated from the weight percentages and not related to any assumptions about molecular weight distributions are the useful and correct parameter which would scale as concentration. Further, it is straightforward to count the number of monomers from the oligomer distributions, since the number of monomers in a particular oligomer is known from the mass. Therefore, again, the concentrations are best directly described as scaled by monomer mole ratios, and not "molar" ratios in the traditional sense of small molecule chemistry. Table 3 lists the calculated total intensity ratios for both PDMS2200 and PDMS6140. As discussed before, the absolute intensities of MALDI strongly depend on the shape and size of the matrix crystals. The absolute intensities measured in the present work vary by up to $30 \%$ (data not shown). That is the major limitation of MALDI for quantitative analysis. Ordinarily an internal standard is needed to normalize the analyte signals. However, the differences in structures and chemical properties between internal standard materials and analyte could still cause different crystallization and hence produce error. PDMS2200 and PDMS6140 share identical chain structures (except chain length), end groups, and similar chemical properties. Therefore, comparison of the MALDI signals between these two polymers could eliminate the uneven crystallization problem and produce more accurate results.

The ratio of total intensity of PDMS2200, $\mathrm{I}_{\mathrm{t} / 2 \mathrm{k}}$, to the total intensity of PDMS6140, $\mathrm{I}_{\mathrm{t} / 6 \mathrm{k}}$, and the ratio of monomer molar concentration of PDMS2200, $\mathrm{M}_{2 \mathrm{k}}$, to the monomer molar concentration of PDMS6600, $\mathrm{M}_{6 \mathrm{k}}$, are listed in Table 3. The measured results are in good agreement (within 2 standard deviation) with the ratios predicted from the stoichiometry. A plot of relative ion intensity ratios (PDMS6140/PDMS2200, as Y axis) as a function of percentage of PDMS6140/PDMS2200 (weight/weight, as $\mathrm{X}$ axis) is shown in Figure 4. The line passes through the origin, with a slope of 0.94 and correlation coefficient $\left(R^{2}\right)$ of 0.99 . The result implies 


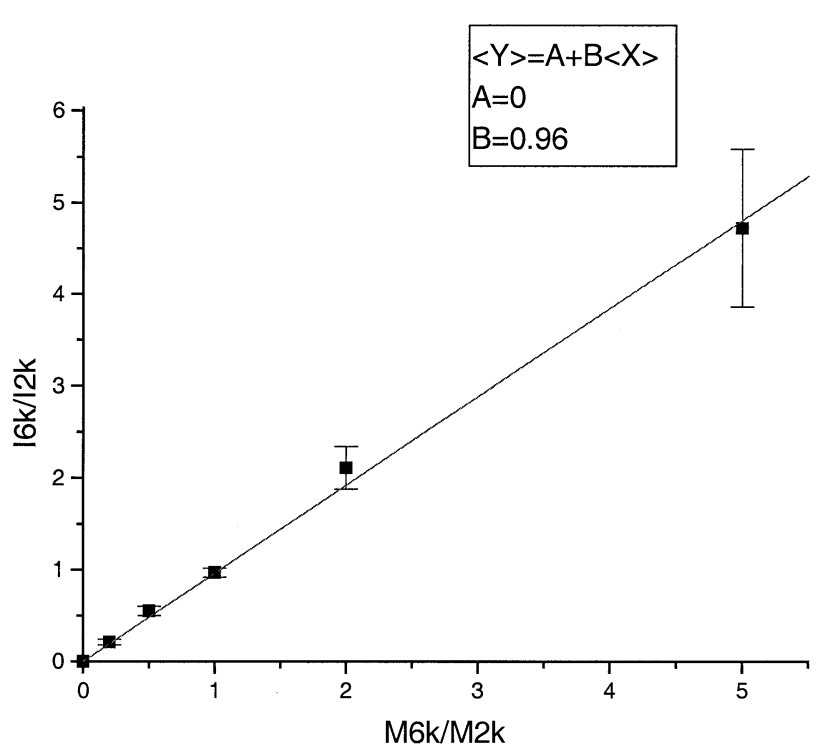

Figure 4. Plot of relative ion intensities ratios versus the weight ratios of polydimethylsiloxane.

that different length polymer chains display equal ionization/desorption probabilities.

In order to study the effect of a third component (PMMA) on the ionization/desorption probabilities, samples from mixture set $B$ were designed to change the percent of PMMA in the mixture, while the relative ratios of PDMS2200/PDMS6140 were the same, as shown in Table 1.

Symmetric Gaussian-like distributions and the same type of three-peak repeat patterns were observed in each spectrum. Eq 4 was used to calculate the total signal intensities of each polymer. The ratio of total intensity of PDMS2200, $I_{t / 2 k}$, to the total intensity of PDMS6140, $I_{t / 6 k}$, and the ratio of molar concentration of PDMS2200, $\mathrm{M}_{2 \mathrm{k}}$ to the molar concentration of PDMS6140, $\mathrm{M}_{6 \mathrm{k}}$, are listed in Table 4.

The MALDI results showed that the ratios of relative signal intensities of PDMS6140 to PDMS2200 were equal to 1 within 2 standard deviation, as the predicted data from polymer concentrations. The presence of PMMA in the mixture did not have an effect on the ionization/desorption probability of either low or high PDMS polymers. Since there was a lack of a PMMA signal, no direct comparison could be made between the PDMS data and the PMMA data.

Table 4. Comparison of signal intensity ratios to concentration ratios

\begin{tabular}{lccc}
\hline Name & $\begin{array}{c}\mathrm{V}_{6140} / \mathrm{V}_{2200} \\
(\mu \mathrm{l})\end{array}$ & $\begin{array}{c}\mathrm{M}_{6140} / \mathrm{M}_{2200} \\
(\text { theoretical) }\end{array}$ & $\begin{array}{c}\mathrm{I}_{\mathrm{t} / 2 \mathrm{k}} / \mathrm{I}_{\mathrm{t} / 6 \mathrm{k}} \\
\text { (calculated) }\end{array}$ \\
\hline \hline Sample 1 & $50 / 50$ & 1.0 & $1.02 \pm 0.11$ \\
Sample 2 & $40 / 40$ & 1.0 & $1.23 \pm 0.07$ \\
Sample 3 & $30 / 30$ & 1.0 & $0.97 \pm 0.07$ \\
Sample 4 & $20 / 20$ & 1.0 & $1.11 \pm 0.07$ \\
Sample 5 & $10 / 10$ & 1.0 & $0.85 \pm 0.05$ \\
Sample 6 & $150 / 150$ & 1.0 & $1.05 \pm 0.14$ \\
\hline
\end{tabular}

\section{Conclusion}

MALDI mass spectrometry was utilized to quantitatively study mixtures of PDMS and PMMA technical polymer systems. The results showed that the different length oligomers of PDMS displayed equal ionization/ desorption probabilities over a range of $1000 \mathrm{Da}$ to $10,000 \mathrm{Da}$, regardless of the presence of a third polymer PMMA. The results also confirmed that MALDI could provide quantitative information of polymer systems with an error less than 2 standard deviation. Based upon published literature, it appears that this work is the first study of the quantitative measurements of synthetic polymer concentration by MALDI.

\section{References}

1. Nelson, R. W.; McLean, M. A. Anal. Chem. 1994, 66, 1408-1415.

2. Muddiman, D. C.; Gusev, A. I.; Proctor, A.; Hercules, D. M. Anal. Chem. 1994, 66, 2362-2368.

3. Nicola, A. J.; Gusev, A. I.; Hercules, D. M. App. Spectros. 1996, 50, 1479-1482.

4. Muddiman, D. C.; Gusev, A. I.; Martin, L. B.; Hercules, D. M. Fresenius J. Anal. Chem. 1996, 354, 455-463.

5. Mehl, J. T.; Nicola, A. J.; Isbell, D. T.; Gusev, A. I.; Hercules, D. M. Am. Lab. 1998, 30, 30-38.

6. Gusev, A. I.; Wilkinson, W. R.; Proctor, A.; Hercules, D. M. Fresenius J. Anal. Chem. 1996, 354, 455-463.

7. Belu, A. M.; DeSimone, J. M.; Linton, R. W.; Lange, G. W.; Friedman, R. M. J. Am. Soc. Mass. Spectrom. 1996, 7, 11-24.

8. Montaudo, M. S.; Puglisi, C.; Samperi, F.; Montaudo, G. Rapid Commun. Mass Spectrom. 1998, 12, 519-528.

9. Aaserud, D.; Prokai, L.; Simonsick, W., Jr. Anal. Chem. 1999, 71, 4793-4799.

10. Wu, J.; Chatman, K.; Harris, K.; Siuzdak, G. Anal. Chem. 1997, 69, 3767-3771.

11. Bornsen, K. O.; Mohr, M. D. Anal. Methods Instr. 1995, 2, 158-160.

12. Vestal, M. L.; Juhasz, P.; Martin, S. A. Rapid Commun. Mass Spectrom. 1995, 9, 1044-1050.

13. Axelsson, J.; Scrivener, E.; Haddleton, D. M.; Derrick, P. J. Macromol. 1996, 29, 8875-8882.

14. Voyager Biospectrometry Workstation User's Guide. PerSeptive Biosystems, Inc.: Framingham, MA.

15. (a) Hawkridge, A. M.; Gardella, J. A., Jr. . Polym. Prepr. Am. Chem. Soc. 2000, 41(1), 635-636. (b) Hawkridge, A. M.; Gardella, J. A. Jr. Macromol., unpublished.

16. Axelsson, J.; Scrivener, E.; Haddleton, D. M.; Derrick, P. J. Macromol. 1996, 29, 8875-8882.

17. Yan, W.; Ammon, D. M., Jr.; Gardella, J. A., Jr.; Maziarz, E. P., III; Hawkridge, A. M.; Grobe, G. L., III; Wood, T. D. Euro. Mass Spectrom. 1998, 4, 467-474.

18. Schriemer, D. C.; Li, L. Anal. Chem. 1997, 69, 4176.

19. Danis, P. O.; Karr, D. E.; Simonsick, W. J., Jr.; Wu, D. T. Macromol. 1995, 28, 1232.

20. Wu, K. J.; Odom, R. W. Anal. Chem. 1998, 70, 458A.

21. Lehrle, R. S., Sarson, D. S. Rapid Commun. Mass Spectrom., 1995, 9, 92.

22. Guttman, C. M.; Wetzel, S. J.; Blair, W. R.; Fanconi, B. M.; Girard, J. E.; Goldschmidt, R. J.; Wallace, W. E.; VanderHart, D. L. Anal. Chem. 2001, 73, 1252-1262.

23. Goldschmidt, R. J. Ph.D. Thesis, Drexel University, Philadelphia, PA. 1998.

24. Chen, R.; Zhang, N.; Tseng, A. M.; Li, L. Rapid Commun. Mass Spectrom. 2000, 14, 2175-2181. 